\title{
Video-based data acquisition system for use in eye blink classical conditioning procedures in sheep
}

\author{
Kelsey Nation ${ }^{1} \cdot$ Adam Birge $^{1} \cdot$ Emily Lunde $^{1} \cdot$ Timothy Cudd $^{1} \cdot$ Charles Goodlett $^{2}$. \\ Shannon Washburn ${ }^{1}$
}

Published online: 4 November 2016

(C) Psychonomic Society, Inc. 2016

\begin{abstract}
Pavlovian eye blink conditioning (EBC) has been extensively studied in humans and laboratory animals, providing one of the best-understood models of learning in neuroscience. EBC has been especially useful in translational studies of cerebellar and hippocampal function. We recently reported a novel extension of EBC procedures for use in sheep, and now describe new advances in a digital video-based system. The system delivers paired presentations of conditioned stimuli (CSs; a tone) and unconditioned stimuli (USs; an air puff to the eye), or CS-alone "unpaired" trials. This system tracks the linear distance between the eyelids to identify blinks occurring as either unconditioned (URs) or conditioned (CRs) responses, to a resolution of $5 \mathrm{~ms}$. A separate software application (Eye Blink Reviewer) is used to review and autoscore the trial CRs and URs, on the basis of a set of predetermined rules, permitting an operator to confirm (or rescore, if needed) the autoscore results, thereby providing quality control for accuracy of scoring. Learning curves may then be quantified in terms of the frequencies of CRs over sessions, both on trials with paired CS-US presentations and on CS-alone trials. The latency to CR onset, latency to CR peak, and occurrence of URs are also obtained. As we demonstrated in two example
\end{abstract}

Electronic supplementary material The online version of this article (doi:10.3758/s13428-016-0826-x) contains supplementary material, which is available to authorized users.

Shannon Washburn

swashburn@cvm.tamu.edu

1 Department of Veterinary Physiology and Pharmacology, College of Veterinary Medicine and Biomedical Sciences, Texas A\&M University, College Station, Texas 77843-4466, USA

2 Department of Psychology, Indiana University-Purdue University Indianapolis, Indianapolis, Indiana, USA cases, this video-based system provides efficient automated means to conduct EBC in sheep and can facilitate fully powered studies with multigroup designs that involve paired and unpaired training. This can help extend new studies in sheep, a species well suited for translational studies of neurodevelopmental disorders resulting from gestational exposure to drugs, toxins, or intrauterine distress.

Keywords Cerebellar-dependent learning - Automated data collection · Pavlovian eye blink conditioning .

Translational neuroscience

Classical conditioning of an eyeblink response (eyeblink conditioning, EBC) is one of the most extensively studied and best-understood models of learning in neuroscience (Woodruff-Pak \& Steinmetz, 2000a, 2000b), with more than 1,200 publications from research in mice, rats, rabbits, and humans addressing its behavioral, developmental, and neurobiological mechanisms. EBC is a form of Pavlovian associative learning in which the subject is given numerous paired presentations of a "neutral" conditioned stimulus (CS) - for instance, a tone that does not cause a reflexive blink - and an unconditioned stimulus (US) - for instance, a puff of air to the cornea of the eye that does cause a reliable blink, called an unconditioned response (UR). Over repeated trials of paired presentations, learning is expressed by the occurrence of an eyeblink response that occurs upon presentation of the CS in advance of (or even in the absence of) the US, termed a conditioned response (CR). The rate of acquisition of this learning and the expression of CRs to CS presentations can be used as indexes of the functional integrity of the neural circuitry involved in EBC.

Decades of research have identified the behavioral properties and the neural circuitry essential for $\mathrm{EBC}$, and extensive 
knowledge of the cellular and molecular mechanisms of learning-related neuroplasticity has developed (Freeman \& Steinmetz, 2011; Kim \& Thompson, 1997; Krupa, Thompson, \& Thompson, 1993; Steinmetz, 2000; Thompson \& Kim, 1996; Thompson \& Steinmetz, 2009). Learning-related neuroplasticity in the cerebellar interpositus nucleus and in portions of the cerebellar cortex that project to the interpositus are required for normal acquisition of such conditioning. The rate of acquisition of standard "delay" conditioning, in which the CS precedes, overlaps, and coterminates with the US, depends on the characteristics of the CS and US (e.g., intensity, salience) and on the interval between the CS and US onsets, with the optimal interval typically ranging between 250 and $650 \mathrm{~ms}$, depending on species and age (Stanton, 2000). Other variations in conditioning procedures, such as imposing a "silent" interval between the offset of the CS and the onset of the US (called trace conditioning), or reversal following discrimination training, can make the acquisition of EBC also depend on additional circuitry in the forebrain, most notably the hippocampal formation. The cerebellum is required for all forms of EBC, typical for most sensorimotor conditioning, but the task variants that additionally require the functional integrity of hippocampal circuits make EBC well suited for a neural-systems analysis of learning and memory (Stanton, 2000).

One of the most useful aspects of EBC is its value for translational studies of clinical and neurodevelopmental disorders that involve disruption of the neural circuits mediating cerebellar-dependent (and hippocampal-dependent) learning (Woodruff-Pak \& Steinmetz, 2000a, 2000b). For example, experimental rodent models of brain damage induced by binge-like alcohol exposure during the period of brain development comparable to that of the human third trimester have shown that EBC was significantly impaired (Green, 2004; Green, Johnson, Goodlett, \& Steinmetz, 2002; Green, Rogers, Goodlett, \& Steinmetz, 2000; Green, Tran, Steinmetz, \& Goodlett, 2002; Stanton \& Goodlett, 1998), and this finding subsequently led to a similar finding in humans with fetal alcohol spectrum disorder (Jacobson et al., 2008). The predictive validity of EBC in various animal models as an indicator of functional damage in underlying neural circuits makes it a powerful behavioral assay to help identify the consequences of (and potential treatments for) neurodevelopmental disorders.

EBC has had wide experimental application across many species, from mice to humans, and we previously published the first EBC study in sheep (Johnson, Stanton, Goodlett, \& Cudd, 2008). Extending EBC methods to sheep is a relevant advancement, because sheep have several unique advantages as an animal model of prenatal development for use in preclinical studies in maternal-fetal medicine. For example, our laboratory has developed the sheep model to study binge-like prenatal alcohol exposure to identify the consequences and potential mechanisms of prenatal brain injury (Cudd, 2005; Sawant, Lunde, et al., 2013; Sawant, Ramadoss, Hankins, Wu, \& Washburn, 2014a, 2014b; Sawant, Ramadoss, Hogan, \& Washburn, 2013; Sawant, Wu, \& Washburn, 2015; Washburn, Ramadoss, Chen, \& Cudd, 2014; Washburn, Sawant, Lunde, Wu, \& Cudd, 2013; Washburn, Tress, Lunde, Chen, \& Cudd, 2013; West, Parnell, Chen, \& Cudd, 2001; Wilson \& Cudd, 2011). As part of this research program, we have developed methods to assess EBC as a postnatal behavioral endpoint in weanling lambs (Johnson et al., 2008).

Since that original study, we have developed a novel digital video-based system for monitoring and recording eye blink activity in the sheep, along with improved, custom software to control the experimental procedures and provide automated measurement, scoring, and analysis of EBC. In the present report, we describe this state-of-the art system in terms of the automated control of stimulus events and tracking and the measurement of eyelid responses to a resolution of $5 \mathrm{~ms}$. A separate software application (the Eye Blink Reviewer) is used to review the trials and autoscore each for the occurrence of CRs and URs, on the basis of a set of predetermined rules, and to provide quality control by the operator. This video-based system provides an advanced means to study EBC in sheep, expanding opportunities to use sheep models in translational studies such as those involving prenatal insults from exposure to drugs, toxins, metabolic disorders, or intrauterine distress that may impact the developing neural systems involved in learning and memory.

\section{Method}

\section{Mask design}

A flexible mask was constructed out of neoprene fabric and industrial strength Velcro, cut to fit the head of a lamb (Fig. 1a). Ear holes were cut to allow for the ears to anchor the mask, eye holes were cut into both sides of the mask, and the mask extended to just above the nose and mouth. A vertical slit was made between the ear holes, which extended approximately $1.5 \mathrm{in}$. from the end of the mask and was secured with Velcro to allow for adjustments to the distance between the ears on different lambs. Additional cuts were made for adjustments to the nose area of the mask, starting at the edges of the mask that sit on the sides of the face and extending approximately $2 \mathrm{in}$. toward the eye holes. The mask is cut lengthwise underneath the chin, and one end is covered in Velcro to adjust the snugness of the entire mask to the head. 


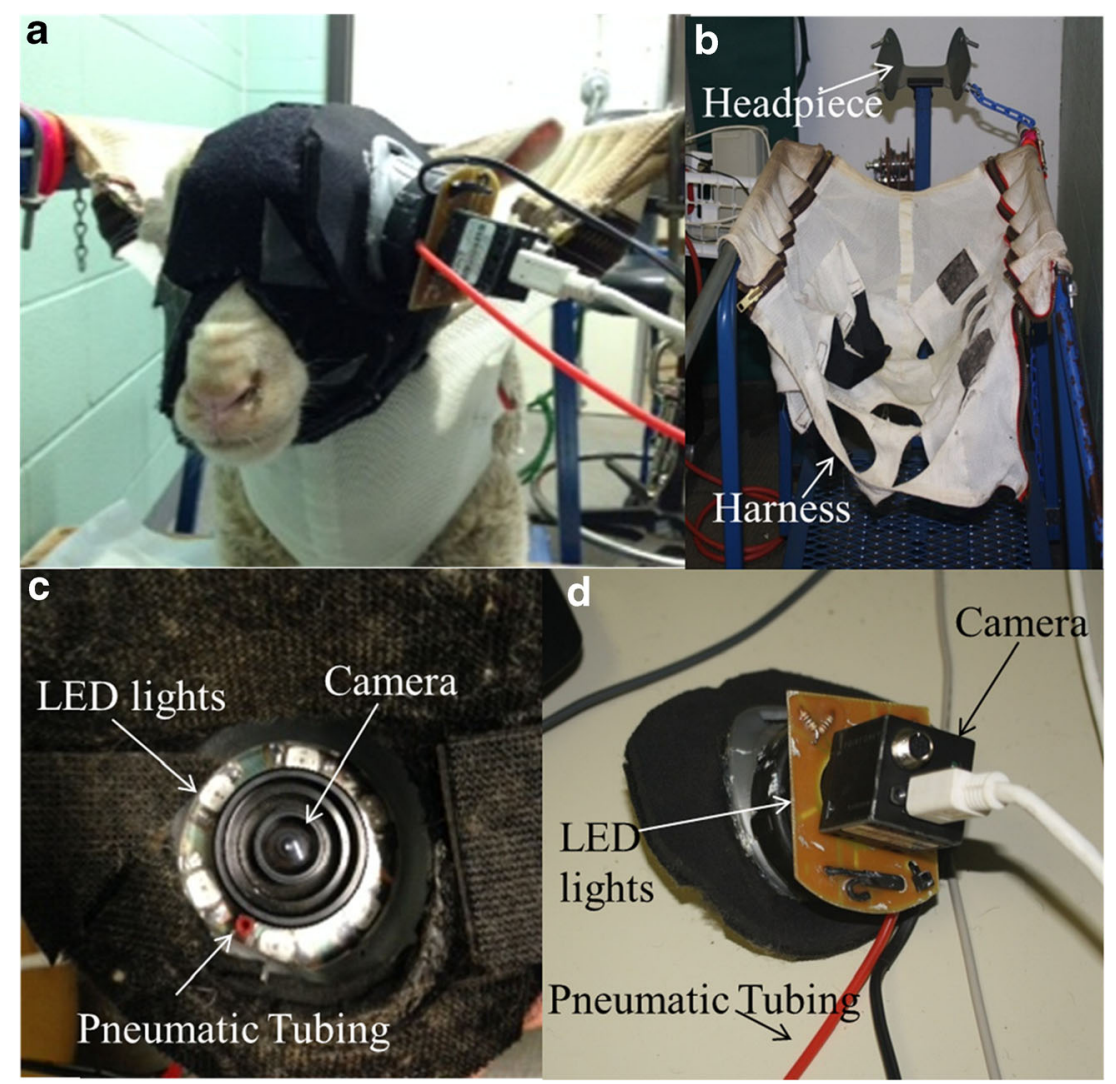

Fig. 1 Components of the materials and peripheral hardware for eye blink conditioning in sheep. (a) Neoprene mask on lamb with goggle and camera attached. (b) Mesh harness and trim stand headpiece. (c)

\section{Lamb restraint}

A mesh harness was used to secure the lambs to the trim stand (Fig. 1b). The lambs were positioned with their legs in the leg holes, and then the harness was pulled snugly against the lamb's body and secured with Velcro along the lamb's back. The lamb was then put onto a trim stand with sides that had been modified to have fabric with zippers attached to it. The harness was zippered along the length of the lamb on both sides to the trim stand.

During the earlier portions of this experiment, a halter was placed on the lamb's head and clipped to cross ties that extended from the sides of the trim stand. The lambs outgrew this design, so during the last week of the experiment a trim stand with a head piece was used instead of crossties (Fig. 1b). A plastic chain around the back of the head was loosely secured to prevent the side of the head piece from pushing against the goggle.

\section{Goggle design}

The lens portion of the camera [FL3 FW-03S1M (IEEE 1394), Point Grey, Canada] was unscrewed from the rest of the camera, and LED lights were placed over the now-exposed threads
Goggle from inside, showing the positions of the camera, pneumatic tubing, and LED lights. (d) Goggle from the outside, with camera, pneumatic tubing, and LED lights

of the camera and faced away from the camera. The left eyepiece of a goggle was converted to fit over the left eye of the lamb. A hole was cut into the center of the goggle lens, and the camera with LEDs was inserted from the outside of the goggle into the hole and secured by screwing the camera lens onto the camera from the inside of the goggle. A second, smaller hole was cut into the goggle, and the pneumatic tubing for delivery of the US was fed into this hole. The hole for the tubing was placed as close to the camera lens as was possible, with the position slightly caudal-ventral to the camera when the goggle was placed over the eye. This positioning ensured that the air puff was delivered to the cornea and was out of the field of view of the camera. The tubing was inserted so that it was extending to the same distance inside of the goggle as the edge of the camera lens (Fig. 1c). Neoprene fabric was glued to the edges of the goggle and extended outward from the goggle in all directions (Fig. 1d). Industrial strength Velcro was cut to fit over the entirety of the neoprene so that the goggle could be secured to the mask.

\section{Eyeblink training procedures}

The software provides precise temporal control over stimulus events (up to two different CS presentations and one US 
presentation for each trial) and tracks the linear distance between the upper and lower eyelids in real time, to a resolution of $5 \mathrm{~ms}$, to identify eye blinks that occur either as URs (blinks after the onset of the air puff) or CRs (blinks after the onset of the tone CS and before the onset of the air puff US). The software system has the flexibility to program multiple different task variants (paradigms) of Pavlovian conditioning, including single-CS conditioning (e.g., "delay" or "trace" conditioning) or multiple-CS conditioning (e.g., discrimination training in which one CS is paired with the US and one is not). For this report, only delay training procedures were evaluated for EBC applications in sheep, using two postweaning lambs; delay eye blink training is typically the most basic and most commonly used procedure in other species.

As is shown in Fig. 2, "short-delay" training consisted of trials (1,000-ms duration) of paired presentations of a whitenoise CS (onset at $250 \mathrm{~ms}$ after the start of each trial; duration of $350 \mathrm{~ms}$ ), followed by an air puff (onset $550 \mathrm{~ms}$ after the start of the trial, 50-ms duration), such that the CS and US overlapped (beginning at $550 \mathrm{~ms}$ ) and co-terminated (at $600 \mathrm{~ms}$ ) after the start of the trial. These parameters yielded an interstimulus interval (the time between the CS onset and the US onset) of $300 \mathrm{~ms}$, which our preliminary data suggested was within the optimal interval for acquisition for lambs. Every 10th trial was a CS-alone (probe) trial, in which the CS presentation (350 ms) was the same but the US was omitted. Two lambs [B75 (male) and W75 (female)] were trained with short-delay procedures, with acquisition first being evaluated at 13 weeks of age with daily training sessions over five consecutive days. Then, after a 7-week hiatus, short-delay retention/reacquisition was assessed beginning at 21 weeks of age, when the two lambs were retrained on the same short-delay paradigm to a criterion of $90 \%$ CRs on two consecutive days.

\section{Scoring of response types}

After training (described below), the sessions are opened in the Eye Blink Reviewer portion of the software. Upon opening a session for the first time in the reviewer, the software processes every trial of the session and generates a summary file of response types and blink measurements.
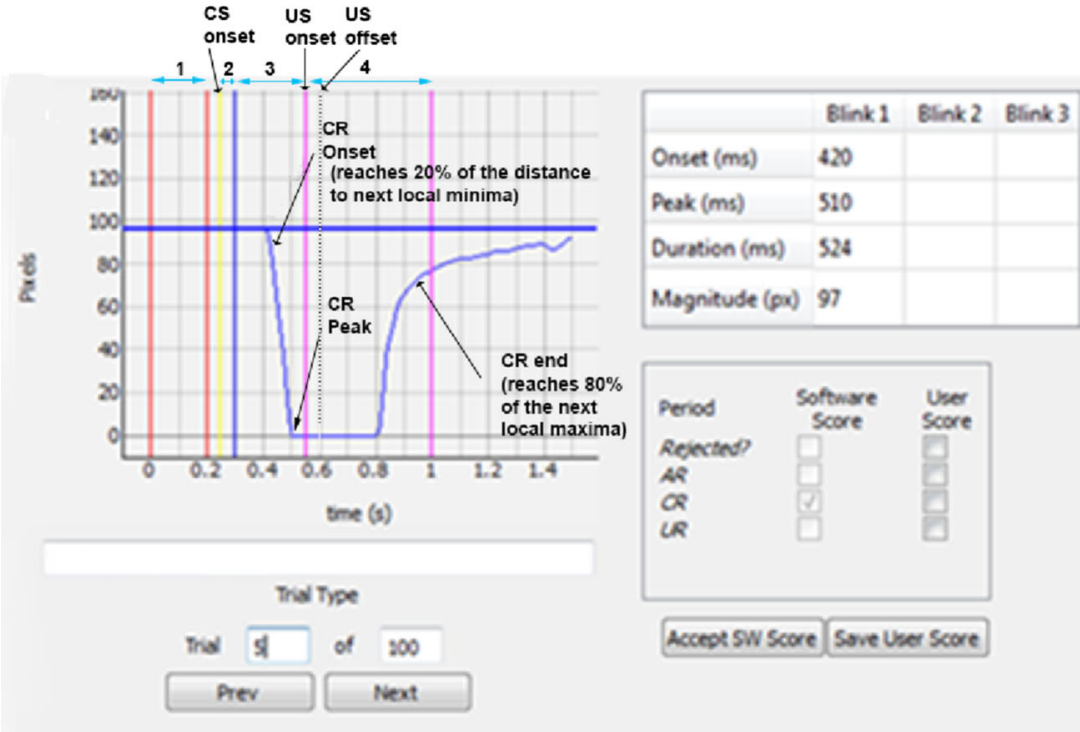

Fig. 2 Eye Blink Reviewer display of the output of a completed delayconditioning trial. Note that the autoscoring indicates a putative CR (Blink 1) with onset at $420 \mathrm{~ms}$ (the point at which the eyelid separation reaches $20 \%$ of the total distance between the most recent "local maximum" [the baseline, in this case], and the next "local minimum" [the point at which the eyelid is completely closed, in this example, with a value of 0]). The autoscoring also identifies the CR peak at 510 $\mathrm{ms}$, with a magnitude of 97 pixels (complete eyelid closure from a baseline of 97 pixels to 0 pixels for this putative CR) and a duration of $524 \mathrm{~ms}$. The annotations depict the timing of the stimulus events and trial epochs. The $x$-axis is the time in seconds, and the $y$-axis is the linear distance between the eyelids, measured in pixels. The baseline period is from the start of the trial until the CS presentation at $250 \mathrm{~ms}$ (the vertical yellow line), and the period labeled "1" (horizontal blue arrows between the two red vertical lines marking the first $200 \mathrm{~ms}$ of the baseline period) is used to establish the stability of the eyelid just prior to stimulus delivery. Any trial with a baseline standard deviation greater than three pixels will be rejected by the autoscore algorithm. The short interval labeled " 2 ," between the vertical yellow line (CS onset) and the vertical blue line (at $300 \mathrm{~ms}$ ), is the AR (startle) period, which extends for $50 \mathrm{~ms}$ after CS onset. The CR response period is labeled " 3 "; it begins right after the AR period and ends with the onset of the air puff US at $550 \mathrm{~ms}$ (marked by the first magenta vertical line). The UR response period, labeled " 4 ," begins with the US onset and extends to the end of the trial at $1,000 \mathrm{~ms}$ (second magenta vertical line). The dark gray vertical dotted line at $600 \mathrm{~ms}$ depicts the co-termination of the tone CS and the air puff US. Note that the blink is scored a putative CR by the software, because its onset was during the $\mathrm{CR}$ period and extended through most of the rest of the trial; no UR is scored because the CR produced a complete eyelid closure that extended through the US delivery and beyond 
Trial inclusion/rejection The baseline period for each trial is defined as the first $250 \mathrm{~ms}$ of the trial (interval before CS presentation), and it is analyzed for stable eyelid recording (assessed during the first $200 \mathrm{~ms}$ of the 250-ms baseline period). The criteria for stability of the eyelid during this period are as follows: (a) standard deviation of the baseline no more than three pixels, and (b) no baseline change more than \pm 3 standard deviation units above/below the mean baseline. Any trials not meeting these criteria are rejected for scoring. Lamb B75 had overall percent rejection rates on paired trials of $20 \%$ (autoscore) and 15\% (rescored), and overall rejection rates of $11 \%$ (autoscore) and 14\% (rescored) for the probe trials. Similarly, W75 had rejection rates of $14 \%$ (autoscore) and $11 \%$ (rescored) for paired trials, and 10\% (autoscore) and $14 \%$ (rescored) for probe trials.

Blink detection The algorithm identifies local minima in the waveform, and a given minimum is considered a blink under the following conditions: (a) the magnitude between onset and peak is at least five pixels (if beyond the baseline period), and (b) there has not been a lower minimum within the preceding $100 \mathrm{~ms}$. For all identified minima occurring after the baseline period, the onset is determined by finding the highest point (local maxima) between the current and the previous minimum (or from $100 \mathrm{~ms}$ into the trial, if this is the first minimum). Onset is determined as the nearest time point after the local maximum at which the eyelid distance reaches $20 \%$ of the total difference between the local and the (subsequent) target minimum. Note that with this approach of determining blinks on the basis of the detection of local minima relative to the just-previous local maxima (with a minimum magnitude of five pixels), multiple eye blinks over a single trial can be automatically identified and scored, including the presence of both a CR and a UR when they occur on a paired trial. For a duration calculation, the "end" of the target blink is when its waveform crosses the $80 \%$ level between the target blink's local minimum and the subsequent local maximum.

The software determines response types from the userestablished parameters for the stimulus events and time periods of response types, from which the software parses each trial into blink activity that occurs during a baseline period, a startle (alpha response) period after CS onset, a CR period, and a UR period. As is depicted in Fig. 2 for a short-delay trial (1,000-ms total duration), the first $250 \mathrm{~ms}$ constitute the baseline period (before CS presentation), and eyelid activity during the first $200 \mathrm{~ms}$ of that period is used to establish the stability of the eyelid prior to stimulus delivery; trials with unstable baselines are rejected for scoring. The CS onset is at $250 \mathrm{~ms}$, and any blinks beginning during the first $50 \mathrm{~ms}(250-300 \mathrm{~ms})$ are recorded as "alpha" responses (AR) - that is, nonassociative startle responses. CRs are scored for blinks that occur between the end of the AR period and the US onset $(301-550 \mathrm{~ms})$. URs can be detected and scored on paired trials for any blink that meets the local minimum criteria occurring after the onset of the air puff US $(550 \mathrm{~ms})$ until the end of the trial $(1,000 \mathrm{~ms})$. For CS-alone trials, only ARs and CRs are scored.

\section{Behavioral training procedures}

\section{Habituation}

Lambs were separated from the ewes at 8 weeks of age and were given two weeks of habituation to the EBC procedures (daily sessions, 5 days a week) beginning at 11 weeks of age. During the first two days, the harness and neoprene mask were put on the lambs, and each lamb was tied by a halter in an area away from the other sheep (10 and $20 \mathrm{~min}$, respectively). On the third through the fifth days, the harness and mask were placed on the lamb, the lamb was placed in the trim stand, and a dummy goggle was attached to the mask $(15,30$, and 45 min, respectively). During the second week of habituation, the lambs were in the stand wearing the harness, mask, and goggle for $20 \mathrm{~min}$ a day. The lamb then underwent five days of shortdelay eye blink training at 13 weeks of age (after the two-week habituation); another week of short-delay retraining was given at 21 weeks of age to assess retention.

\section{Eye blink training}

Lambs were placed on the trim stand and restrained as described above with the harness, mask, goggle, and head restraint. For all procedures described above, a white-noise CS of approximately $75 \mathrm{~dB}$ was projected from a PC speaker placed approximately 2 feet in front of and to the left of the lamb. The ambient noise levels were approximately $60 \mathrm{~dB}$. The US was an air puff (source pressure 15 psi, solenoid model 6200-0001-F, San Diego Instruments, San Diego, CA; with a solenoid opening time of $10-20 \mathrm{~ms}$ ), directed at the cornea (the air pressure delivered to the cornea at the end of the tube was not determined). The US was controlled by a solenoid and air pressure regulator that was interfaced with the training software and connected to a pressured nitrogen tank. The time for the solenoid to open upon command was nearly instantaneous, but the exact time required for the air column to reach the cornea after the command signal was generated was not determined. Each training session consisted of 100 trials and generally lasted for approximately $45 \mathrm{~min}$. Every tenth trial was a CS-alone probe trial. The intertrial intervals were randomly generated and lasted from 5 to $15 \mathrm{~s}$.

\section{Performing a session}

The graphical display window depicts the events and sampling periods of the trial epochs and is labeled. For all trial types (CS1, CS2, or probe), the screen depicts and labels all 


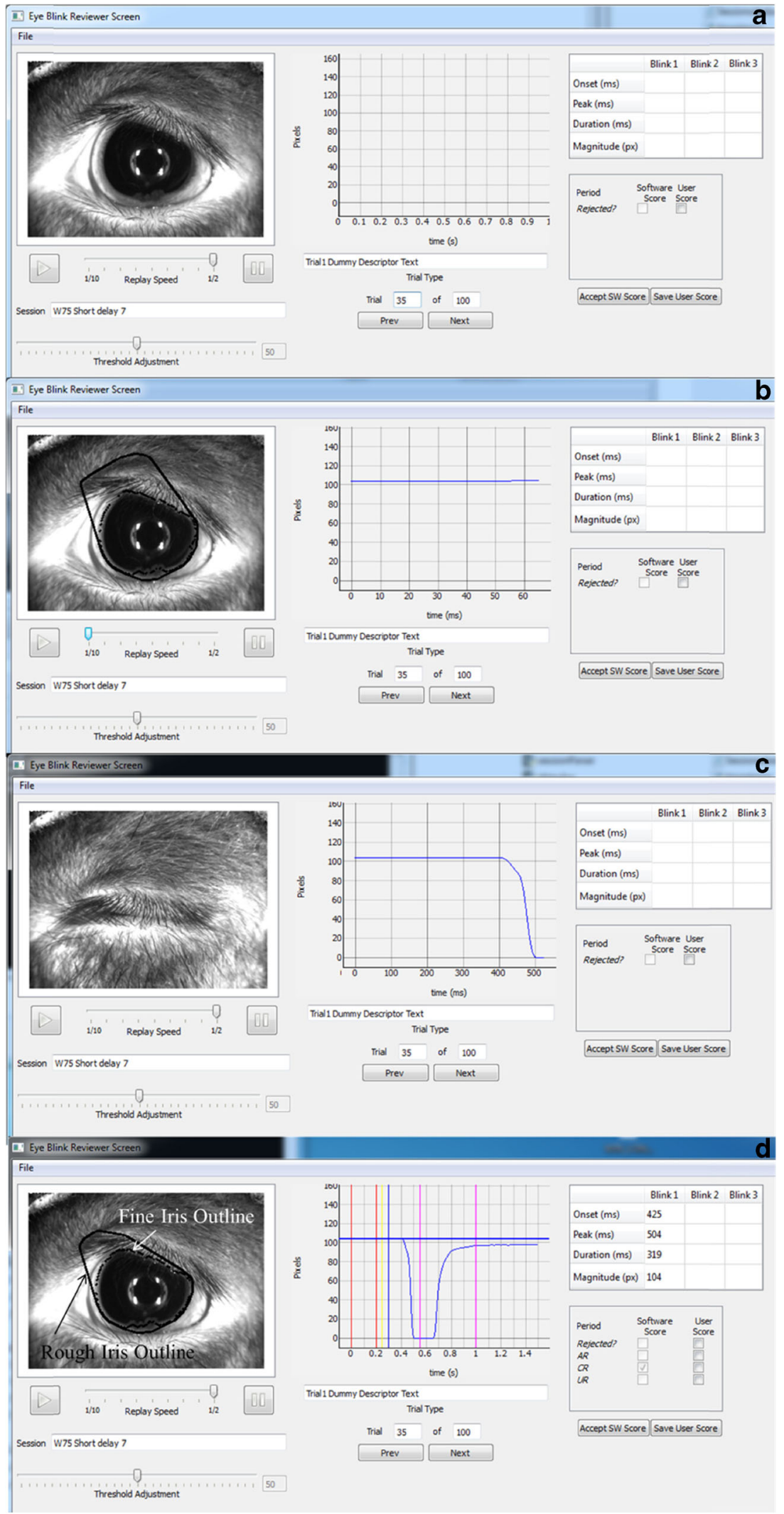

epochs, onsets, and offsets of each stimulus. The $x$-axis is the duration of the trial (defined in the session descriptor file); the $y$-axis is determined by the baseline magnitude and the linear difference between the upper and lower eyelids when the eye 
4 Fig. 3 Eye Blink Reviewer, showing a time series of still frames of a short-delay paired trial (35th trial of Training Day 7). (a) The trial is loaded and ready to play. The video is on the left and the graphing area is in the center. Underneath the video player is a slider bar for adjusting the replay speed between $1 / 10$ and1/2 of the actual speed of the trial, along with "Play" and "Pause" buttons. Underneath the "Play" button is the current session being reviewed, and beneath that is a threshold adjustment bar for altering the iris tracking. Underneath the graph is a text box for the user to enter comments, as well as the current trial number and buttons to move back or forward a trial. The user can also type the trial number that he or she would like to go to and press Enter to move between trials within a session. (b) The "Play" button has been pressed, and the trial has begun replay (for about $65 \mathrm{~ms}$ ). The user has adjusted the replay speed to $1 / 10$ th to take a screenshot. The solid black line is the rough iris outline, and the dotted black line inside the solid black line is the fine iris outline, which are both updated with every video frame. The graph is generated as the video is playing so that the user can match what is seen on the video to the time point. (c) The trial replay (just past 500 $\mathrm{ms}$ ) is at the peak of a blink that is a putative CR with its onset at $425 \mathrm{~ms}$. As the eye closes, the linear distance within the iris outline decreases. At this time point the eye is completely closed (the only "local minimum" in this trial), with no detectable iris, so the pixel measurement has gone to zero. (d) After the trial replay has finished, the vertical lines that appear on the graph mark the user-inputted parameters for scoring the response types. In this short-delay example, the graph shows lines for the beginning of the trial (left red line), the end of the baseline period (red line at $200 \mathrm{~ms}$ ), the CS onset (yellow line at $250 \mathrm{~ms}$ ), the border between $\mathrm{AR}$ and $\mathrm{CR}$ responses (blue line at $300 \mathrm{~ms}$ ), the border between $\mathrm{CR}$ and UR responses (magenta line at $550 \mathrm{~ms}$ ), and the end of the UR scoring period (magenta line at 1,000 ms). On the upper right, the information about the timing of the blink is recorded. If multiple blinks have occurred, the data will be recorded for each of the first three blinks. On the lower right, the software checks the box(es) for the response types that occurred, and the user has the option of saving the software score to indicate that the trial has been reviewed, or to input a new score if he or she disagrees with the software

is open. The "Time to Next Trial" box depicts seconds before the next trial (the number of seconds between trials is randomly selected on the basis of a range defined in the session descriptor file); the "Trials Completed" box depicts the number of trials completed and the number remaining. The "Trial Type" box indicates a brief description for the particular paradigm, as defined in the session descriptor file.

The "Play" button allows the user to connect to the camera and stream live video prior to the session starting, to ensure that the mask is properly in place. The user starts a new session and is prompted to choose the applicable session descriptor file and a directory for the results (raw video files and scoring output) to be placed in. The session is started by choosing the "Record" button. Once the session has started, all of the trials are automatically run, and the "Trials Completed" window informs the experimenter how many trials have been completed. For each trial, the time to next trial counts down, the trial starts (i.e., image collection begins), the CS is administered, the US is administered in paired trials (no US is delivered in probe trials), and the trial is completed (image collection ends). Immediately at the end of a trial, the trial video and graph are displayed simultaneously and synchronized in time in slow motion, allowing the operator to see the corresponding eye blink and the timing of the events, allowing for provisional manual scoring by the experimenter. Once in progress, the session can be paused between trials to readjust the camera equipment, or stopped to abort the session.

When a session is started, a randomized number of seconds will occur between trials, and all of the trials will be run automatically until the session is complete. For a CS1 trial, at the end of the baseline a white-noise tone is delivered at the CS1 onset and terminated at the CS1 offset. An air puff, the US, is delivered at the US onset and terminated at the US offset. For $\mathrm{CS} 2$ trials (discrimination training) the trial begins, and at the end of the baseline, the CS2 is activated at CS2 onset through a USB switch. CS2 is then turned off at CS2 offset. For a probe trial, at the end of the baseline, CS1 onset is activated and no US is presented. Raw video files (.rvf) are written as they are completed, trial by trial. All of the data from a session are placed in a user-selected folder that contains one .rvf file for each trial and the eye blink session descriptor file that defines the session. This folder and the files will later be utilized by the Eye Blink Reviewer program. The session can be paused and unpaused using the "Pause" button. The "Stop" button terminates the session, but all data are retained.

\section{Eye blink reviewer program: autoscoring and operator quality control}

The Eye Blink Reviewer program (Fig. 3) is utilized independent of the data collection. In the reviewer program, the operator selects the session folder or individual trial that he or she wishes to review. When reviewing an entire session, the operator can have the program create a .csv summary file, which will be saved to the session folder and will contain the eye blink scores and blink parameter data. When loading a session, Trial 1 is queued up and a " 1 " is entered in the "Current Trial" box. The first time that the reviewer program is loaded, it will automatically run rapidly through each trial to generate the autoscore. Once an autoscore has been generated, the user can review each trial independently. The operator pushes the "Run" button, and the trial then runs in slow motion and the autoscore is displayed. The speed is variable, from $1 / 2$ to 1/10th speed, with $1 / 2$ set as the default. During playback, for each frame the software tries to determine and overlay the outline of the iris, and the eye blink data are generated as the maximum linear distance between the upper and lower eyelids. The operator can observe the simultaneous and synchronized presentation of the video and the charting of the eye blink data graphically. The final chart identifies the CS onset, CS offset, US onset, and US offset, as well as the baseline, AR, CR, and UR periods. The operator can repeatedly review the trial. Any individual trial video contents can also be exported to a standalone avi file. 
Autoscore chooses and displays "AR," "CR," "UR," or "Rejected" according to the algorithmic rules described above. A no-response can also occur if no blink is recorded in any of the response periods. The operator can manually rescore trials if he or she disagrees with the autoscore, ensuring quality control by manually rejecting individual trials for scoring due to baseline instability, excessive head or eye movement during the trial, or lost video signal. During manual review, the operator can select either "Accept SW Score" or "Save User Score." If the operator chooses "Accept SW Score," the summary file will update to show that the user has reviewed that trial. If the operator chooses "Save User Score" the summary file will update to show that the trial was scored by the operator, and the recorded score will be changed to the score that was manually inputted by the operator.

The iris detection algorithm is designed to discern between the iris and the surrounding frame (eyelids). Due to lighting differences and animal appearance, a single threshold for the binary filter may not be optimal in all cases. The "Threshold Adjustment" slider bar can be adjusted by the operator on a trial-by-trial basis to compensate for these differences, and the autoscore will adjust on the basis of the new parameter. The operator can replay the trial and observe the overlaid output from the iris detection algorithm for feedback. The threshold value chosen by the operator will be saved with the results. Upon approval, the next trial number is then automatically entered into the "Current Trial" box. The operator may move between trials by clicking "Prev" or "Next," or by directly typing in a trial number to jump to. For each trial, up to the first three blinks that meet the scoring requirements are recorded. For each blink, the time of onset (in milliseconds), the time when the peak change in eyelid distance occurred (in milliseconds), the maximal magnitude (distance in pixels), and the duration of each blink (in milliseconds) are displayed. On the basis of the time of onset, the blink will be scored in one of the defined periods. The CR, UR, and AR data are evaluated separately from one another for each trial.

The "Trial Type" box is updated for each reviewed trial. Vertical lines are displayed in the graphical display to indicate the scoring periods for the trial. The scoring periods for each trial type are created by the operator in the session descriptor file (period name, start and end times).

Figure $3 \mathrm{~d}$ shows what is displayed in the Eye Blink Reviewer for a typical paired short-delay trial, depicting the distance between the eyelids ( $y$-axis) over time ( $x$-axis). For scored trials, an eye blink CR is identified when the onset of a blink (onset of the local minimum; see above) is identified after CS onset (vertical blue line in Fig. 3d) and before the US onset (first vertical magenta line in Fig. 3d). A UR is scored when the onset of a blink (onset of the local minimum; see above) occurs after the US onset. A blink is classified as an AR if the blink occurs (onset of local minimum; see above) during the AR period (between the yellow and blue vertical lines in Fig. 3d). A trial is excluded if the standard deviation of the baseline data exceeds three pixels or if any baseline deflection exceeds three standard deviations, either of which may occur when a blink onset happens during the baseline period or when instability may result from excessive head/eye movement during baseline. For a CR, the amplitude is recorded as the maximum difference between the local maximum and minimum identifying the blink (or the value at the time of US onset). If the eye blink paradigm includes a second CS (i.e., a CS2 onset and offset), then the timing of these events and the resulting CRs could also be so identified.

Artifacts in video tracking of the eyelid distance, and resulting errors in the automated EBC scoring, could occur due to interruption of detection of the video-based signal used by the software to discern between the iris and the surrounding frame (eyelid detection). An example of such an interruption can be seen in Fig. 4, in which the video detection of the circular pattern of the LED lights on the iris (left panel) was lost when the animal rotated its eye inward and forward (right panel). This resulted in loss of the video signal needed for establishing the eyelid boundaries and loss of integrity of the signal used to determine the eyelid separation. Typically, interruption of the signal that lasted beyond the limits of correction by the threshold adjustment would result in a rejected trial.

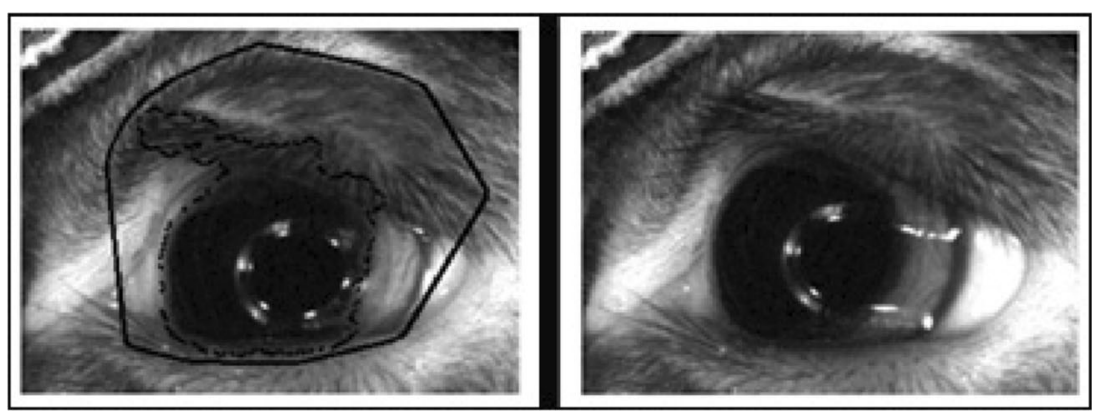

Fig. 4 Example of a trial with a tracking artifact that prevents proper scoring by the computer. (a) The LED lights and camera are centered over the lamb's eye at the beginning of the trial, and the rough and fine iris outlines are present. (b) Reflection and loss of tracking due to forward and inward eye roll. The outlines are lost because the iris cannot be identified 


\section{Results}

Figure 5 shows the acquisition curves of two lambs [B75 and W75], depicting the percentages of scored trials in which eye blink responses occurred during the $\mathrm{CR}$ period (putative CRs), showing both the autoscored outcomes (dashed lines) and the outcomes after experimenter review (solid lines) using the Eye Blink Reviewer. Note the high degree of correlation between the autoscored and the session-reviewed data $(r=.923)$. Both of the lambs given short-delay training acquired eye blink responding during the $\mathrm{CR}$ period over the course of the initial five-day training period at 13 weeks of age, and they demonstrated rapid reacquisition of the putative CRs eight weeks later. Note that lamb B75 showed relatively high rates of eye blink responding even on the first day of training (66\% during the $\mathrm{CR}$ period), and even on the first block of nine paired trials it emitted eye blinks during the CR period on $15 \%$ of the trials, then on $>65 \%$ of the remaining scored trials on the first day. Lamb B75 also reacquired high response rates in the CR period at 21 weeks old, meeting the criterion of $>90 \%$ putative CRs on two consecutive days by the third day of reacquisition training. Lamb W75 showed a more typical acquisition curve, emitting very few CRs on the first day, but reaching $80 \%$ CRs by the third day of training. Lamb W75 showed substantial savings during the first two days of reacquisition when retrained at 21 weeks of age. Video examples of paired trials showing the dynamics of the conditioned eye blinks for lamb W75 can be seen in the video files "W75session5trial5pairedCR" and "W75session7trial35pairedCR," available in the supplemental materials.

The topography (timing and magnitude) of the putative CRs across acquisition training is best assessed on probe trials, in which no US is presented, thus permitting analysis of the full expression of any emitted eye blinks. Acquisition of the putative CRs expressed on probe trials is shown in terms of

\section{Short Delay Acquisition: Paired Trials}

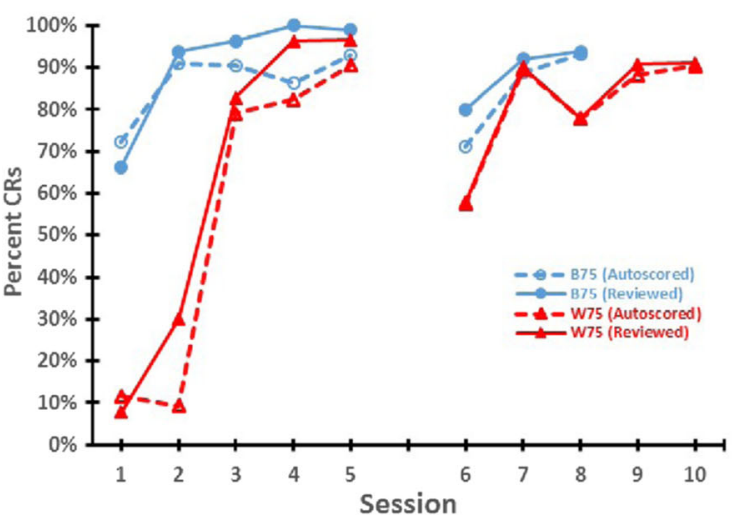

Fig. 5 Acquisition of eye blinks occurring during the CR period (putative CRs) by two lambs given short-delay training procedures, showing the computer-autoscored and the experimenter-reviewed results for both lambs. The first five sessions (one session per day) were at 13 weeks of age; Sessions 6-10 (also one session per day) were at 21 frequency in Fig. 5 (right panel), and in a graphic depiction of the selected probe trials across training in Fig. 6, and corresponding dynamic video records of those trials can be found in in files "W75Ssession 2 trial70probeCR," "W 75 S s e s sion 2 tria 1100 probe C R, "a nd "W75Ssession5trial100probeCR." Note that across training, the increase in the frequency of putative CRs on probe trials was also associated with growth in magnitude of the eye blink response, from its absence on Training Day 1 (0 pixels) to magnitude means that were $>75$ pixels by Training Day 3 .

The autoscoring software provides data that have a high degree of accuracy, and the added quality control function of the Eye Blink Reviewer allows correction of any apparent misclassifications of eyelid activity. The overall agreement between the autoscored and experimenter-reviewed CR data was high. Across 1,260 paired trials that were carefully scored by an expert observer and classified as either having or not having a putative CR, the autoscore had a "false positive" putative CR rate of $2.5 \%$ and a "false negative" rate of $9.6 \%$. One source of "false negative" putative CR classifications between the autoscored and the experimenter-reviewed rescored data was in the designation of trials to be rejected (due to instability or artifact in the video tracking of eyelid distance). The software tended to reject some trials that upon review could be kept (and may have contained a putative CR). For example, for Lamb B75 the autoscore rejected a total of 134 trials across the eight sessions, as compared to 108 after experimenter review; for Lamb W75, the totals (across the ten sessions) were 112 versus 102. Across sessions, the percentages of paired trials rejected after session review were 15\% (B75) and 11\% (W75). The primary reason for rejected trials was tracking instability due to movements of the head and eyes (see Fig. 4). We determined that the extensive habituation used for these two lambs was important to limit intrusions of movement, but other factors, such as uncontrolled environmental noise or brief periods of

\section{Short Delay Acquisition: Probe Trials}

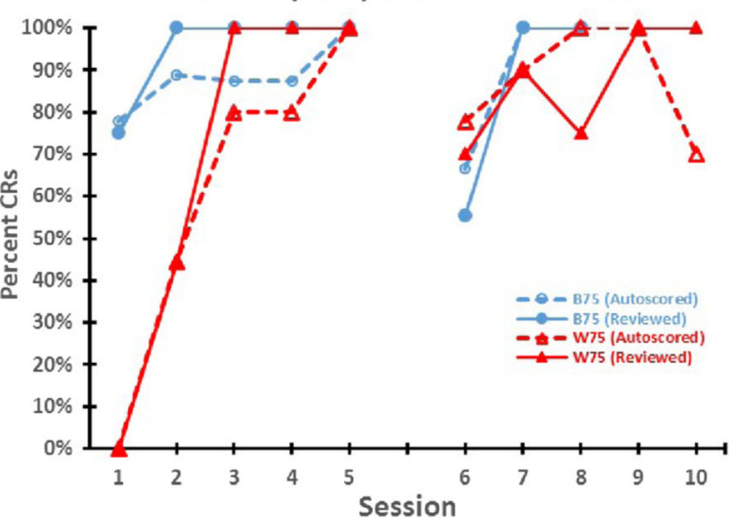

weeks of age (to reacquisition to a criterion of $>90 \%$ two days in a row for Lamb B75, or to five additional days of training for Lamb W75). The left panel shows the paired trials ( 90 trials per session), and right panel shows the CS-alone probe trials (ten probe trials per session) 


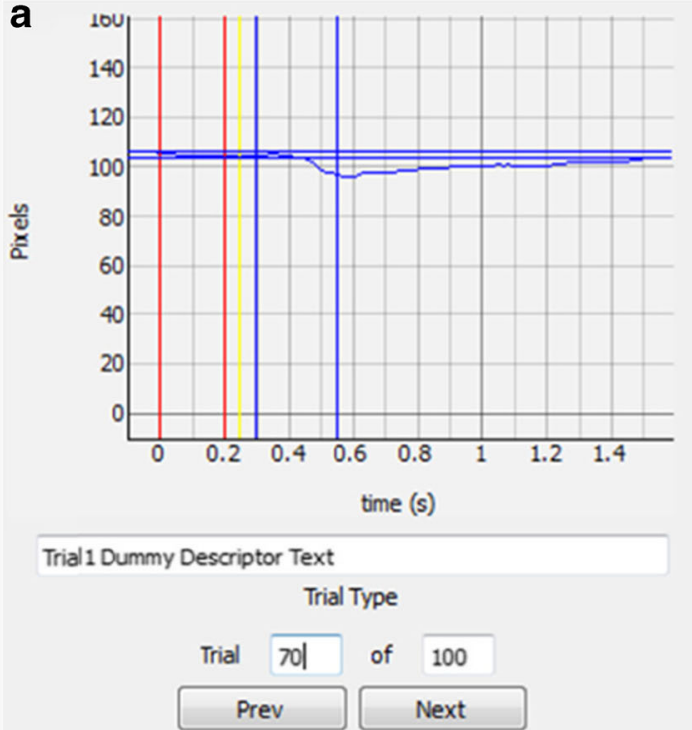

\begin{tabular}{|l|l|l|l|}
\hline & Blink1 & Blink2 & Blink3 \\
\hline Onset (ms) & 450 & & \\
\hline Peak (ms) & 595 & & \\
\hline Duration (ms) & 449 & & \\
\hline Magnitude (px) & 9 & & \\
\hline
\end{tabular}
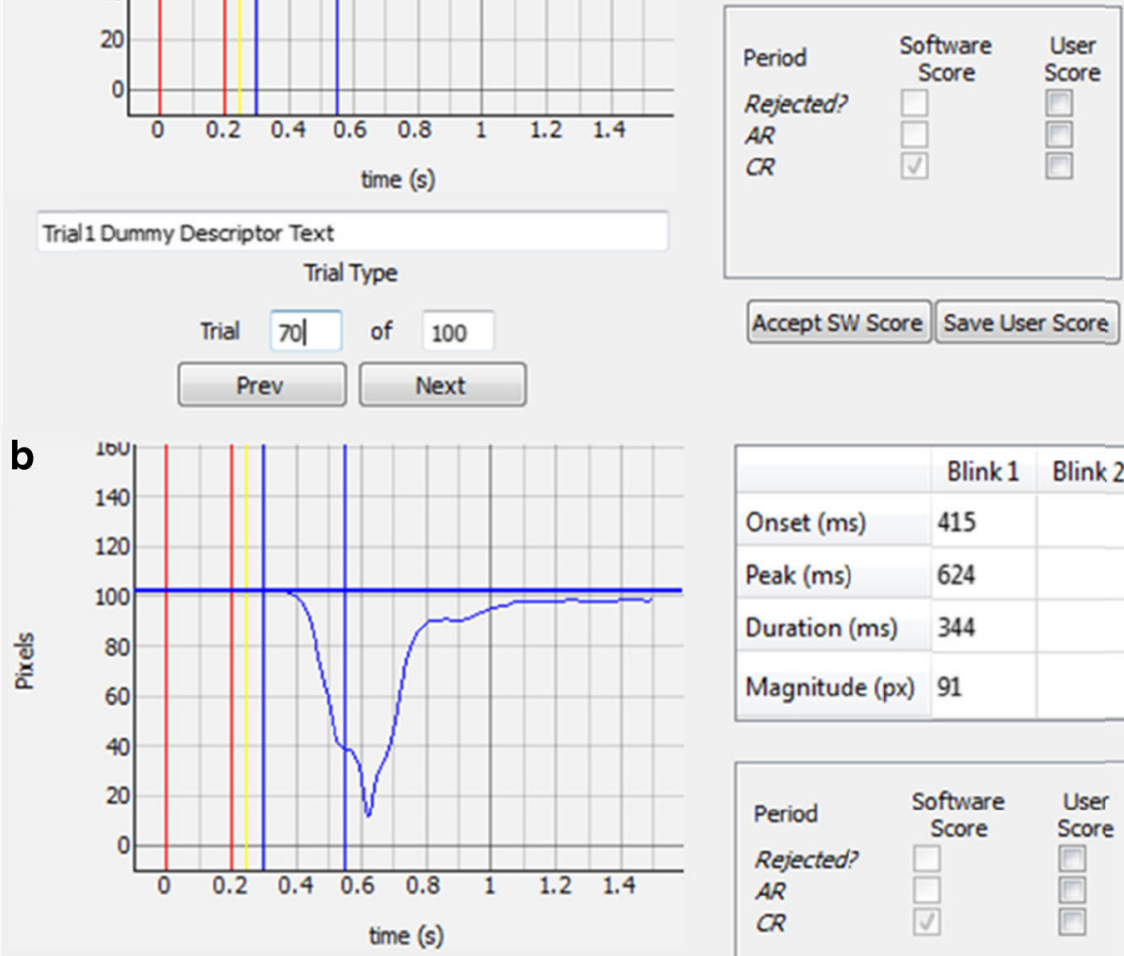

Accept SW Score Save User Score

\begin{tabular}{|l|l|l|l|}
\hline & Blink1 & Blink 2 & Blink 3 \\
\hline Onset (ms) & 415 & & \\
\hline Peak (ms) & 624 & & \\
\hline Duration (ms) & 344 & & \\
\hline Magnitude (px) & 91 & & \\
\hline
\end{tabular}

Some string here...
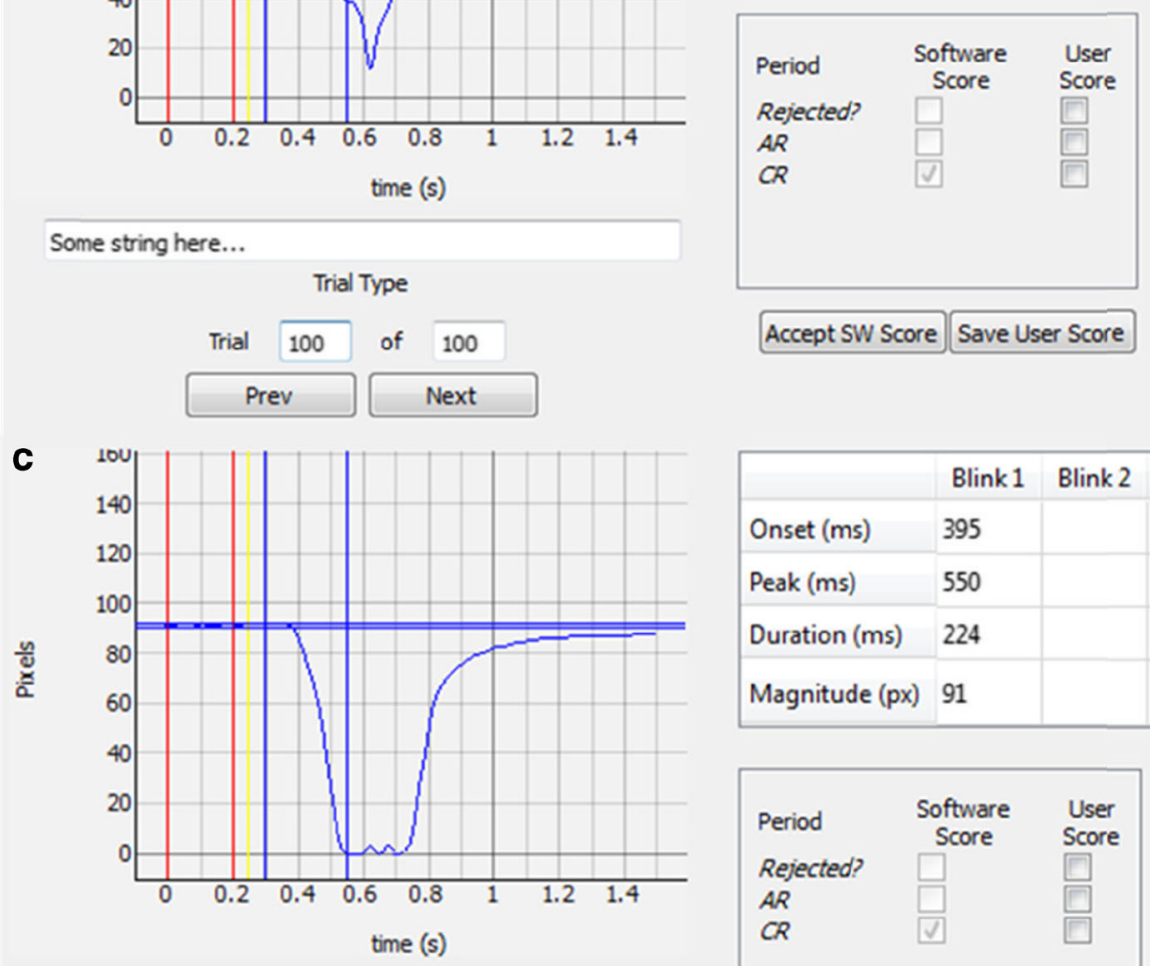

\begin{tabular}{|l|l|l|l|}
\hline & Blink1 & Blink 2 & Blink 3 \\
\hline Onset (ms) & 395 & & \\
\hline Peak (ms) & 550 & & \\
\hline Duration (ms) & 224 & \\
\hline Magnitude (px) & 91 & & \\
\hline
\end{tabular}

Some string here...

Trial Type

\begin{tabular}{|c|c|} 
Trial 100 & of 100 \\
\hline Prev & Next \\
\hline
\end{tabular}

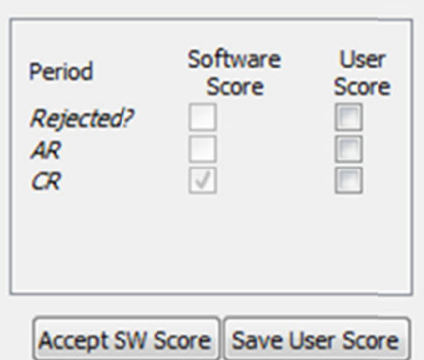


Fig. 6 Progression of the acquisition of putative CRs shown on probe (CS-alone) trials (Lamb W75). (a) A putative CR representative of the short-delay probe trials early in learning (Training Day 2, Trial 70), showing an eye blink with a small magnitude. The peak occurs after the US onset would have occurred in a paired trial, indicated by the line at 550 ms. (b) Putative CR slightly later in training (Training Day 2, Trial 100). The CR amplitude is large, but the peak still occurs after US onset would have occurred. (c) Putative CR from the last probe trial of the first five sessions (Training Day 5, Trial 100). The amplitude is large and the peak occurs at the time point of US onset in paired trials

subjective restlessness of the lamb, could contribute. The ability to temporarily pause the programmed training procedures during such periods until the lamb has settled down helps limit such intrusions. Overall, the autoscore combined with Eye Blink Reviewer provides an accurate and efficient means to obtain reliable quantitative data concerning the acquisition of putative EBC in lambs.

The onset times of the first-occurring blinks across trials showed training-related patterns that were consistent with the acquisition of CRs. During the initial stages of training, the

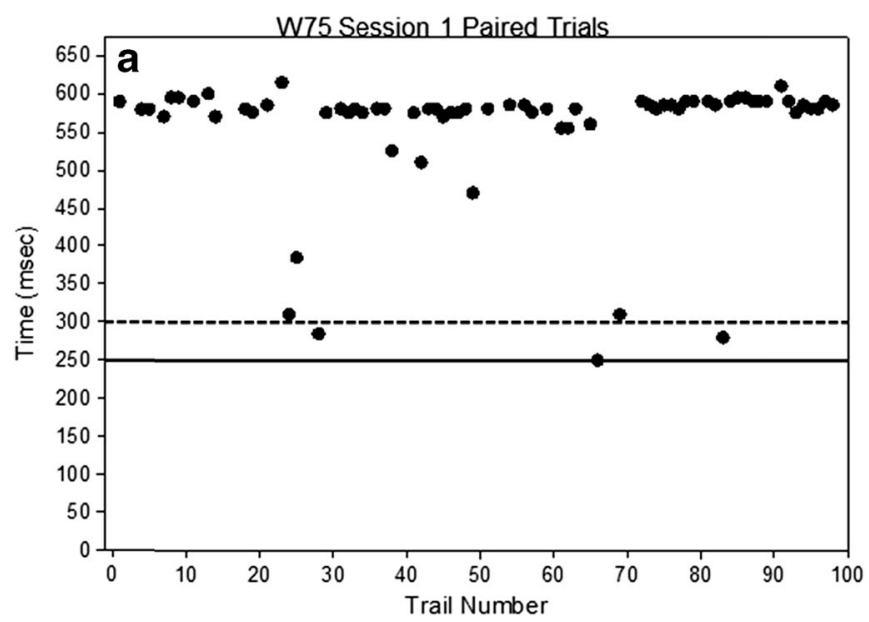

onset of the first blinks occurred mainly after the US presentation (URs), or on rare occasions as alpha responses (startle responses just after CS presentations). As can be seen in Fig. 7, scatterplots of the times of onset of the first blinks across trials for Sessions 1 and 5 of Lamb W75 clearly show a lack of putative CRs (and preponderance of URs) on Session 1, and the preponderance of putative CRs by Session 5 .

\section{Discussion}

The technology and procedures presented here provide the first video-based automated data acquisition system for EBC studies that are devised specifically for sheep, but the software and technology can be adapted to other species, including humans. In terms of experimental control systems, all of the information related to the design of a session is programmed into a text file that is loaded through the Eye Blink Data Acquisition screen. With the beginning of the first trial, video

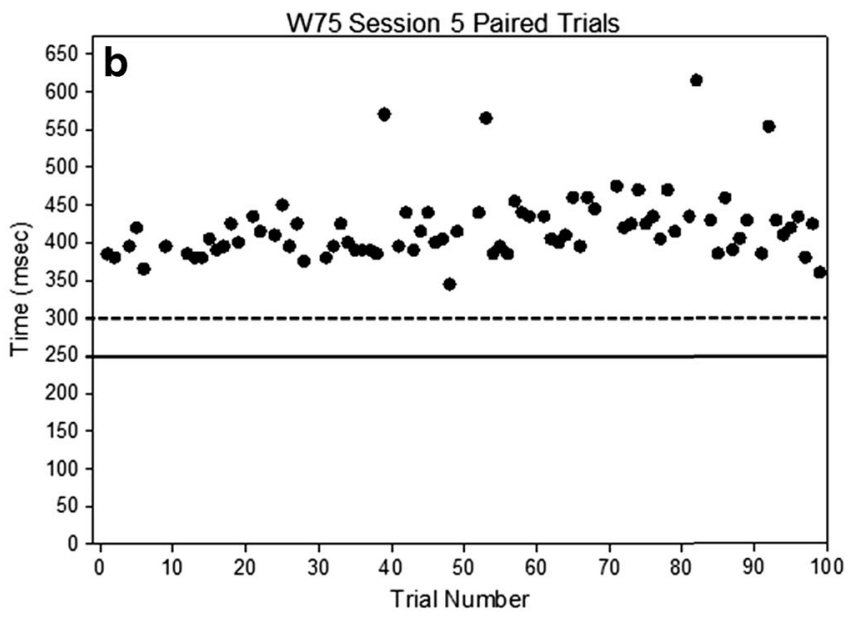

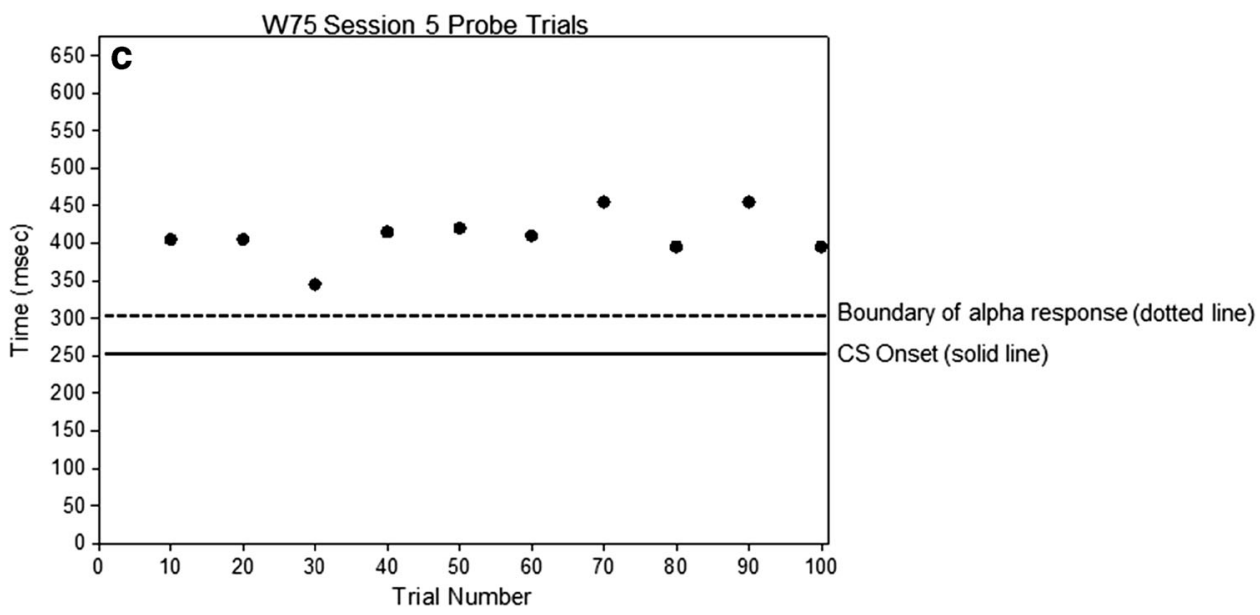

Fig. 7 Plots of trial number ( $x$-axis) versus blink onset time (i.e., the first blink detected; $y$-axis) for Lamb W75: (a) Session 1 paired trials. (b) Session 5 paired trials. (c) Session 5 probe trials. The boundary of the alpha response is depicted with a dashed lines, and the CS onset is depicted as a solid line. These scatterplots reveal not only the (relatively rare) onset times of alpha responses, but also the training-related changes in onset times, showing the acquisition of putative CRs 
images of the eye are acquired at $200 \mathrm{~Hz}$ - that is, one video frame every $5 \mathrm{~ms}$. The software automatically identifies the eyelid margins and calculates a distance between the lids every $5 \mathrm{~ms}$ over the duration of the trial. The video images and eyelid distance data are logged as they are acquired. Immediately following the trial, the video images and eyelid distances (graphed over time) are presented simultaneously and synchronously to the experimenter in slow motion. The software then automatically processes all of the session trials one by one while the experimenter observes, and quality control can be governed during this review by the experimenter.

This report demonstrates an application of video-based technology that will facilitate extension of eye blink conditioning studies to sheep. The two cases presented here, though limited, point to some additional issues and choices about the specific procedural parameters to be used with sheep, which need to be evaluated further and guided by the extensive experimental literatures on other species. For example, the relatively short intertrial intervals used in this demonstration (5$15 \mathrm{~s}$, mean of $10 \mathrm{~s}$ ), and the resulting relatively high density of trials per session (100 trials in about $40 \mathrm{~min}$ ), may not have been optimal for the acquisition of CRs, relative to procedures that use longer ITIs or more distributed training (i.e., fewer trials per session). Previous work in rabbits has shown that intertrial intervals that averaged $9 \mathrm{~s}$ would not support conditioning (Nordholm, Lavond, \& Thompson, 1991). Our initial choice of having shorter ITIs was based on the goal of having sessions with 100 total trials that did not extend more than 35$45 \mathrm{~min}$, to avoid the increasing restlessness of lambs when sessions were longer than $45 \mathrm{~min}$. Nevertheless, the relatively high density of trials with mean ITIs of $10 \mathrm{~s}$ may not be optimal for acquisition by sheep. Additionally, Kehoe and Gormezano (1974) showed that having fewer training trials distributed over more daily sessions produced the fastest learning (in terms of trials to criterion). Further exploration of the conditioning parameters could determine whether having fewer trials per session (e.g., 60-80 trials) with longer average ITIs (e.g., mean of 20-30 s) might better optimize acquisition rates while maintaining session lengths of $\sim 30$ min or less.

The main purpose of this report is to describe and demonstrate the advances made in using video-based technology to study eye blink conditioning in sheep. We recognize that the two lambs used here to demonstrate the functionality of the system do not constitute a definitive demonstration of the associative-learning process involved in eye blink conditioning in lambs. Such evidence would require the inclusion of groups with sufficient numbers of lambs randomly assigned to either a "paired training" group or an "explicitly unpaired" group (that would control for potential pseudoconditioning). The system described here would facilitate such experimental studies of basic delay conditioning, and it could be used for many other useful variants of eye blink training, as well. The system software was designed to provide flexibility to control multiple conditioning paradigms that afford the capability to probe the functional integrity of multiple brain systems involved in learning and memory. This is an important issue, for example, when the research questions emerging from studies of prenatal insults need to address the specificity of the brain injury involved.

The EBC procedures demonstrated here can independently assess sensory functions (e.g., startle blink responses, responses to air puff presentations alone), motor functions (the topography of spontaneous blinks or of unconditioned blink responses), along with the main goal of assessing the neural systems of associative learning, requiring functional integrity of the cerebellar circuitry (delay EBC). The maximum utility of this system, beyond the scope of this demonstration, will come from the capability of programming many other Pavlovian task variants to address questions about the contributions of various additional neural systems involved in "higher-order" forms of learning, including trace conditioning, discrimination training and reversal, latent inhibition (stimulus preexposure), conditioned inhibition, blocking effects, or second-order conditioning.

The potential to assess discrimination learning and reversal involving multiple CSs is an example of the flexibility of the system. During initial discrimination training, sessions would include the presentation of two different CSs, randomly distributed across trials, so that half of the trials would have the first CS (CS1) paired with the air puff US, and the other half would have the second (different) CS (CS2) occurring alone (not paired with a US). After the acquisition of the discrimination to a learning criterion, the associations of the two CSs could be reversed, such that CS1 would now occur without the air puff, whereas CS2 would now be paired with the air puff. This paradigm could be particularly useful when assessing the potential dissociation of hippocampal-dependent deficits (reversal learning) from cerebellar-dependent processes (acquisition of the initial discrimination; Stanton, 2000).

Adapting such procedures to sheep would expand the potential value of various sheep models to include experimental analyses of learning and memory, a significant advantage because of the unique advantages that sheep models provide in studies of maternal-fetal medicine, reproductive physiology and toxicology, and the developmental neuroscience of neurodevelopmental disorders. This is the first system to provide an efficient means to conduct EBC in sheep, a species especially well-suited for translational studies of neurodevelopmental disorders resulting from gestational exposure to drugs, toxins, nutritional deficits, or intrauterine distress.

In particular, sheep models provide key advantages for use in preclinical studies in maternal-fetal medicine (especially as compared to the commonly used rodent species), due to the relatively close match between sheep and humans in terms of the timing and stages of prenatal development relative to birth, 
along with maternal and fetal size over gestation. These advantages particularly apply to experimental studies of neuroteratogenic effects of prenatal insults, which can vary both in duration and in timing across different stages of brain development over the 40-week gestation in humans. Sheep have a relatively long length of gestation ( $\sim 147$ days/21 weeks), and the different phases of prenatal brain development are relatively extended (as in humans). This provides longer windows of time for experimental manipulations, and better comparative matches to the specific timing and duration of prenatal insults in humans. Most critically, unlike most laboratory rodents, the brain developmental processes of all three trimester-equivalents of human pregnancy occur in utero in sheep. The human third trimester includes the so-called "brain growth spurt," the period of peak velocity of accelerated brain growth marked by extensive synaptogenesis and myelination (Dobbing \& Sands, 1973, 1979), and this is a period of vulnerability to a variety of teratogens. The "brain growth spurt" occurs in utero in the sheep, as in humans and unlike in rats and mice, in which it occurs postnatally (Dobbing \& Sands, 1973, 1979). Thus, the sheep model includes all of the prenatal maternal-fetal components across all three trimesterequivalents (maternal physiology, intrauterine environment, placental function, and parturition) necessary to translate experimental findings directly from animal model to human (Cudd, 2005; Wilson \& Cudd, 2011).

Several commercial and custom-made systems are available for eye blink conditioning in humans and rodents. The versions for humans (Cheng, Faulkner, Disterhoft, \& Desmond, 2010) typically use infrared sensors to detect eyelid closure for recording eye blinks, deliver air puffs as the USs, and incorporate computer-controlled delivery of the stimuli and recording of the eye blink responses. The system reported here for sheep is conceptually the same, but technically it is based on high-speed video rather than infrared sensors, and the video records of eye blinks on each trial are stored and available for viewing (unlike with the human systems that rely on infrared-based sensors). The systems for mice and rats are typically customized to monitor the electromyographic (EMG) activity of the orbicularis muscle that controls the eye blink, which is quantitative and correlated with the dynamics of eye blinks in those species, and use mild electrical stimulation caudal to the orbit to elicit unconditioned eye blinks (Skelton, 1988; Tran, Jackson, Horn, \& Goodlett, 2005). To the extent that the video-based monitoring measures the eyelid closure directly - rather than the EMG activity associated with eye blinks - the measures obtained in sheep are more akin to those reported in human studies.

Some nuances of the topography of the sheep eye blink measure with direct video detection make the measurement of blink responses somewhat different from the EMG measures of rodent eye blink responses. A good example is evident in the video for Lamb W75, Session 7 Trial 35. As can be seen there, eyelid closure occurs with the initial blink, but a second, strong muscle contraction then occurs while the eyelid is still closed; this second movement (perhaps a form of a "double blink") occurs while the eyelid waveform tracing is still at zero (at floor), because the eyelid is still closed. This second component is not identified as a second blink, though it can contribute to extending the duration of the blink, which is measured by the software analysis program. It should be noted that EMG-based detection systems typically are capable of resolving multiple contractions of the orbicularis muscle because the EMG signal does not saturate with the initial blink. Nevertheless, video-based technology has been used effectively in previous EBC applications in mice (Siegel et al., 2015) and rabbits (Bracha, Nilaweera, Zenitsky, \& Irwin, 2003), and has been adapted for supplemental measures in humans (Claflin, Stanton, Herbert, Greer, \& Eckerman, 2002; Kwon et al., 2013). Despite potential species differences in eye blink response topography and the means of measuring those eye blinks across species and platforms, one of the key aspects of eye blink conditioning is the high degree of convergence of the behavioral and neurobiological findings.

The development of this system provides a new tool to permit assessment of multiple forms of learning and memory in sheep that involve different underlying neural systems (e.g., cerebellar-dependent and hippocampal-dependent learning). Analysis of EBC also provides the capability of identifying treatment effects that specifically target the neural mechanisms of associative learning and memory and separate them from effects on the sensory or motor components of the behavior. This automated system to study EBC in sheep has the potential to open new opportunities to use ovine models, which have many advantages in modeling prenatal insults in humans that alter brain development, to include advanced and specific assessment of learning and memory that can be directly translated to humans.

Author note We thank Dillon Johnson, Sarah Baker, and Lance Wheeler for their assistance. Supported by NIH Grant Nos. U01 AA17120 and 18166-2 (to S.W.).

\section{References}

Bracha, V., Nilaweera, W., Zenitsky, G., \& Irwin, K. (2003). Video recording system for the measurement of eyelid movements during classical conditioning of the eyeblink response in the rabbit. Journal of Neuroscience Methods, 125, 173-181.

Cheng, D. T., Faulkner, M. L., Disterhoft, J. F., \& Desmond, J. E. (2010). The effects of aging in delay and trace human eyeblink conditioning. Psychology and Aging, 25, 684-690. doi:10.1037/a0017978

Claflin, D. I., Stanton, M. E., Herbert, J., Greer, J., \& Eckerman, C. O. (2002). Effect of delay interval on classical eyeblink conditioning in 5-month-old human infants. Developmental Psychobiology, 41, 329-340. doi:10.1002/dev.10050 
Cudd, T. A. (2005). Animal model systems for the study of alcohol teratology. Experimental Biology and Medicine, 230, 389-393.

Dobbing, J., \& Sands, J. (1973). Quantitative growth and development of human brain. Archives of Disease in Childhood, 48, 757-767.

Dobbing, J., \& Sands, J. (1979). Comparative aspects of the brain growth spurt. Early Human Development, 3, 79-83.

Freeman, J. H., \& Steinmetz, A. B. (2011). Neural circuitry and plasticity mechanisms underlying delay eyeblink conditioning. Learning and Memory, 18, 666-677. doi:10.1101/lm.2023011

Green, J. T. (2004). The effects of ethanol on the developing cerebellum and eyeblink classical conditioning. Cerebellum, 3, 178-187. doi:10.1080/14734220410017338

Green, J. T., Johnson, T. B., Goodlett, C. R., \& Steinmetz, J. E. (2002). Eyeblink classical conditioning and interpositus nucleus activity are disrupted in adult rats exposed to ethanol as neonates. Learning and Memory, 9, 304-320. doi:10.1101/lm.47602

Green, J. T., Rogers, R. F., Goodlett, C. R., \& Steinmetz, J. E. (2000). Impairment in eyeblink classical conditioning in adult rats exposed to ethanol as neonates. Alcoholism: Clinical and Experimental Research, 24, 438-447.

Green, J. T., Tran, T., Steinmetz, J. E., \& Goodlett, C. R. (2002). Neonatal ethanol produces cerebellar deep nuclear cell loss and correlated disruption of eyeblink conditioning in adult rats. Brain Research, 956, 302-311.

Jacobson, S. W., Stanton, M. E., Molteno, C. D., Burden, M. J., Fuller, D. S., Hoyme, H. E., \& Jacobson, J. L. (2008). Impaired eyeblink conditioning in children with fetal alcohol syndrome. Alcoholism: Clinical and Experimental Research, 32, 365-372. doi:10.1111/j.15300277.2007.00585.x

Johnson, T. B., Stanton, M. E., Goodlett, C. R., \& Cudd, T. A. (2008). Eyeblink classical conditioning in the preweanling lamb. Behavioral Neuroscience, 122, 722-729. doi:10.1037/0735-7044.122.3.722

Kehoe, E. J., \& Gormezano, I. (1974). Effects of trials per session on conditioning of rabbits nictitating-membrane response. Bulletin of the Psychonomic Society, 4, 434-436.

Kim, J. J., \& Thompson, R. F. (1997). Cerebellar circuits and synaptic mechanisms involved in classical eyeblink conditioning. Trends in Neurosciences, 20, 177-181.

Krupa, D. J., Thompson, J. K., \& Thompson, R. F. (1993). Localization of a memory trace in the mammalian brain. Science, 260, 989-991.

Kwon, K. A., Shipley, R. J., Edirisinghe, M., Ezra, D. G., Rose, G., Best, S. M., \& Cameron, R. E. (2013). High-speed camera characterization of voluntary eye blinking kinematics. Journal of the Royal Society Interface, 10, 20130227. doi:10.1098/rsif.2013.0227

Nordholm, A. F., Lavond, D. G., \& Thompson, R. F. (1991). Are eyeblink responses to tone in the decerebrate, decerebellate rabbit conditioned responses? Behavioural Brain Research, 44, 27-34.

Sawant, O. B., Lunde, E. R., Washburn, S. E., Chen, W. J., Goodlett, C. R., \& Cudd, T. A. (2013). Different patterns of regional Purkinje cell loss in the cerebellar vermis as a function of the timing of prenatal ethanol exposure in an ovine model. Neurotoxicology and Teratology, 35, 7-13. doi:10.1016/j.ntt.2012.11.001

Sawant, O. B., Ramadoss, J., Hankins, G. D., Wu, G., \& Washburn, S. E. (2014a). Effects of L-glutamine supplementation on maternal and fetal hemodynamics in gestating ewes exposed to alcohol. Amino Acids, 46, 1981-1996. doi:10.1007/s00726-014-1751-x

Sawant, O. B., Ramadoss, J., Hankins, G. D., Wu, G., \& Washburn, S. E. (2014b). Effects of L-glutamine supplementation on maternal and fetal hemodynamics in gestating ewes exposed to alcohol. Amino Acids, 46, 1981-1996. doi:10.1007/s00726-014-1751-x

Sawant, O. B., Ramadoss, J., Hogan, H. A., \& Washburn, S. E. (2013). The role of acidemia in maternal binge alcohol-induced alterations in fetal bone functional properties. Alcoholism: Clinical and Experimental Research, 37, 1476-1482. doi:10.1111/acer.12118

Sawant, O. B., Wu, G., \& Washburn, S. E. (2015). Maternal L-glutamine supplementation prevents prenatal alcohol exposure-induced fetal growth restriction in an ovine model. Amino Acids, 47, 11831192. doi:10.1007/s00726-015-1945-x

Siegel, J. J., Taylor, W., Gray, R., Kalmbach, B., Zemelman, B. V., Desai, N. S., Chitwood, R. A. (2015). Trace eyeblink conditioning in mice is dependent upon the dorsal medial prefrontal cortex, cerebellum, and amygdala: Behavioral characterization and functional circuitry. eNeuro, 2. doi:10.1523/ENEURO.0051-14.2015

Skelton, R. W. (1988). Bilateral cerebellar lesions disrupt conditioned eyelid responses in unrestrained rats. Behavioral Neuroscience, $102,586-590$.

Stanton, M. E. (2000). Multiple memory systems, development and conditioning. Behavioural Brain Research, 110, 25-37.

Stanton, M. E., \& Goodlett, C. R. (1998). Neonatal ethanol exposure impairs eyeblink conditioning in weanling rats. Alcoholism: Clinical and Experimental Research, 22, 270-275.

Steinmetz, J. E. (2000). Brain substrates of classical eyeblink conditioning: A highly localized but also distributed system. Behavioural Brain Research, 110, 13-24.

Thompson, R. F., \& Kim, J. J. (1996). Memory systems in the brain and localization of a memory. Proceedings of the National Academy of Sciences, 93, 13438-13444.

Thompson, R. F., \& Steinmetz, J. E. (2009). The role of the cerebellum in classical conditioning of discrete behavioral responses. Neuroscience, 162, 732-755. doi:10.1016/j.neuroscience.2009.01.041

Tran, T. D., Jackson, H. D., Horn, K. H., \& Goodlett, C. R. (2005). Vitamin $\mathrm{E}$ does not protect against neonatal ethanol-induced cerebellar damage or deficits in eyeblink classical conditioning in rats. Alcoholism: Clinical and Experimental Research, 29, 117-129.

Washburn, S. E., Ramadoss, J., Chen, W.-J. A., \& Cudd, T. A. (2014), Effects of all three trimester moderate binge alcohol exposure on the foetal hippocampal formation and olfactory bulb. Brain Injury, 29, 104-109. doi:10.3109/02699052.2014.947629

Washburn, S. E., Sawant, O. B., Lunde, E. R., Wu, G., \& Cudd, T. A. (2013). Acute alcohol exposure, acidemia or glutamine administration impacts amino acid homeostasis in ovine maternal and fetal plasma. Amino Acids, 45, 543-554. doi:10.1007/s00726-012-1453-1

Washburn, S. E., Tress, U., Lunde, E. R., Chen, W. J., \& Cudd, T. A. (2013). The role of cortisol in chronic binge alcohol-induced cerebellar injury: Ovine model. Alcohol, 47, 53-61. doi:10.1016/j. alcohol.2012.10.004

West, J. R., Parnell, S. E., Chen, W. J., \& Cudd, T. A. (2001). Alcoholmediated Purkinje cell loss in the absence of hypoxemia during the third trimester in an ovine model system. Alcoholism: Clinical and Experimental Research, 25, 1051-1057.

Wilson, S. E., \& Cudd, T. A. (2011). Focus on: The use of animal models for the study of fetal alcohol spectrum disorders. Alcohol Research and Health, 34, 92-98.

Woodruff-Pak, D. S., \& Steinmetz, J. E. (2000a). Animal models (Vol. 2). Boston: Kluwer.

Woodruff-Pak, D. S., \& Steinmetz, J. E. (2000b). Applications in humans (Vol. 1). Boston: Kluwer. 